\title{
STABILITY AND TRANSPARENCY OF DELAYED BILATERAL TELEOPERATION WITH HAPTIC FEEDBACK
}

\author{
EdGAR ESTRADA ${ }^{a}, \mathrm{WEN} \mathrm{YU}^{a, *}$, XIAOOU LI ${ }^{b}$ \\ ${ }^{a}$ Department of Automatic Control \\ CINVESTAV-IPN, Av IPN 2508, 07360 Mexico City, Mexico \\ e-mail: yuw@ctrl.cinvestav.mx \\ ${ }^{b}$ Department of Computation \\ CINVESTAV-IPN, Av IPN 2508, 07360 Mexico City, Mexico
}

\begin{abstract}
Haptic guidance can improve control accuracy in bilateral teleoperation. With haptic sensing, the human operator feels that he grabs the robot on the remote side. There are results on the stability and transparency analysis of teleoperation without haptic guidance, and the analysis of teleoperation with haptic feedback is only for linear and zero time-delay systems. In this paper, we consider more general cases: the bilateral teleoperation systems have time-varying communication delays, the whole systems are nonlinear, and they have force feedback. By using the admittance human operator model, we propose a new control scheme with the interaction passivity of the teleoperator. The stability and transparency of the master-slave system are proven with the Lyapunov-Krasovskii method. Numerical simulations illustrate the efficiency of the proposed control methods.
\end{abstract}

Keywords: teleoperation, force control, stability, transparency.

\section{Introduction}

Teleoperation systems have become an extensive and interesting field for researchers in the last decade, with the ability to operate from a remote location as the main function of teleoperation systems. It has widespread applications in many areas such as space mission, undersea exploration, hazardous environment, tele-surgery, etc. (Nohmi, 2003; Hokayem and Spong, 2006; Jordan and Bustamante, 2007; Kawashima et al., 2008; Erden and Mari, 2011; Ehrampoosh et al., 2013).

$$
\mathbf{u}_{i}=\frac{1}{k_{i}} \sum_{j \in N_{i, k}}\left(x_{j}-x_{i}\right)
$$

The teleoperation system is a two-port structure as shown in the Fig. 11. It is commonly composed of five elements: the human operator, the master manipulator, the communication channel, the slave manipulator and the environment. If the forces generated by the contact between the slave manipulator and the environment are reflected or transmitted back to the master as a part of

* Corresponding author

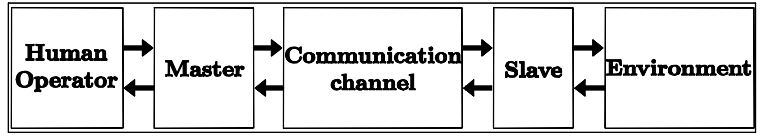

Fig. 1. Two-port structure of the bilateral teleoperation systems.

its input torque, this teleoperation system is bilateral (Anderson and Spong, 1989). The communication delay is a major issue in bilateral teleoperation systems, which may induce instability (Anderson and Spong, 1989; Hokayem and Spong, 2006). Transparency is another challenge in bilateral teleoperacion. The transparency means the operator appropriately feels as if he is manipulating the remote object directly.

In bilateral teleoperation there are two major goals: stability and transparency. Achieving these goals generally improves the operator's ability to perform complex tasks. There are diverse control approaches to reach them. Over the past two decades, the researchers paid more and more attention to develop effective control schemes to solve the first issue: closed-loop stability in the 
bilateral teleoperation systems. One of the most important work is that the bilateral teleoperation systems are stable under arbitrary bounded constant time delay (Anderson and Spong, 1989). They used the transmitting scattering variables rather than the conventional power variables. A similar result proposed by Niemeyer and Slotine (1991) used wave variables to examine the dynamic property of a time delay teleoperation system. Under the framework of passivity based control, some further results have been presented to deal with the problems of time-varying delays (Niemeyer and Slotine, 1998; Yokokohji et al., 1999; Chopra et al., 2003). Munir (2002; 2003) used predictors to compensate the unfavorable effects generated by the time delay, such that higher dynamic performance be obtained. The high-frequency contact forces between the environment and the human operator were transmitted to upgrade transparency of the teloperator in the work of Tanner and Niemeyer (2005).

There are several problems in the above results, such as the wave reflection and the position drift in the passivity based or scattering based approach proposed by Niemeyer and Slotine $(1991 ; 2004)$ as well as Chopra et al. (2006). They induced the packet loss in an unreliable communication network or the time-varying communication delay. The wave reflection problem can be decreased via enforcing impedance matching or adding a wave filter in the communication channel (Niemeyer and Slotine, 1991; 2004). The position drift problem can be alleviated by transmitting both the wave variable and its integral (Niemeyer and Slotine, 1998; 2004), or incorporated the master-slave position tracking errors into the master-slave control inputs (Chopra et al., 2006; 2008; Imaida et al., 2004; Lee and Spong, 2006; Nuno et al., 2008). Recent results show that the synchronization based approach can fundamentally solve the wave reflection and position drift problems that appears in the traditional scattering variable based approach (Imaida et al., 2004; Lee and Spong, 2006; Chopra et al., 2008; Nuno et al., 2008).

There are some methods using control theory. The Lyapunov-based approach can simplify the design process (Imaida et al., 2004; Lee and Spong, 2006). The transparency can be obtained by controller design, see the works by Lawrence (1993) or Yokokohji and Yoshikawa (1994), where the four-channel framework was proposed such that ideal transparency can be attained without any time delay in the communication channel. In order to avoid measuring acceleration in the four-channel control algorithm, Zhu and Salcudean (1995), Salcudean et al. (2000), and Mobasser and Hashtrudi-Zaad (2008) proposed position and rate control algorithms, at the expense of realizing sublevel transparency.

However, all the above papers do not consider haptic sensing in bilateral teleoperation. Without haptic guidance, it is very difficult to perform a precise teleoperation by the human operator. Impedance/admittance control is one of the most effective human-robot integration strategy (Wen and Murphy, 1991). Admittance defines a dynamic mapping from force to motion. An admittance device would sense the input force and "admit" a certain amount of motion. The admittance model from the human operator can be used to replace the master manipulator model in the local site. The object of the bilateral teleoperation with haptic feedback is the following: the robot manipulator and the human operator always have the same position and contract force. The human operator should feel that he grips the end-effector while he handles the teloperator,

The traditional control schemes do not have force controllers that allow a good interaction with the environment in hard contact. An improvement in the position tracking accuracy might give rise to larger contact forces, this problem disrupts the transparency of the closed-loop system during contact motion (Lee and Spong, 2006). The force control (Chiaverini et al., 1994) or the position tracking with impedance models (Nuno et al., 2008; Nuno and Basanez, 2009) can solve the above problems. Here the force control schemes require the dynamics and the inverse dynamics of robots (Nuno et al., 2010). Some control schemes were designed to enhance system transparency by using measured force signals in the control structure (Cho and Park, 2005; Ganjefar et al., 2017; Ishii and Katsura, 2012; Xu et al., 2016; Ousaid et al., 2015). On the other hand, recent works based on the passivity approach, controlling the energy flow at the input/output port are conservative (Rebelo and Schiele, 2015; Chen et al., 2018; Balachandran et al., 2016; Liu et al., 2018). In such cases, they just consider the stability and transparency to linear bilateral teleoperation systems.

But these position-force controllers do not have the analysis of the stability and transparency of nonlinear delayed bilateral teleoperation. In fact, there are special problems in the delayed bilateral teleoperation with haptic feedback. Force control could improve the interaction effect with the environment and avoid large contact forces. The stability is to guarantee stable transition contact. Thus we should transmit the force in conjunction with position signals to enhance a higher transparency.

In this paper, we propose a new control framework for a bilateral teloperator with time-varying communication delays. We include the human operator admittance model in the bilateral teleoperation system. Since the robotic dynamic is nonlinear, we analyze the stability and transparency of the nonlinear teleoperation systems.

The paper is organized as follows. Section 2 introduces the novel bilateral teleoperation system, which combines the human operator model and the delayed bilateral teleoperation model. Section 3 provides stability analysis with the Lyapunov-Krasovskii 


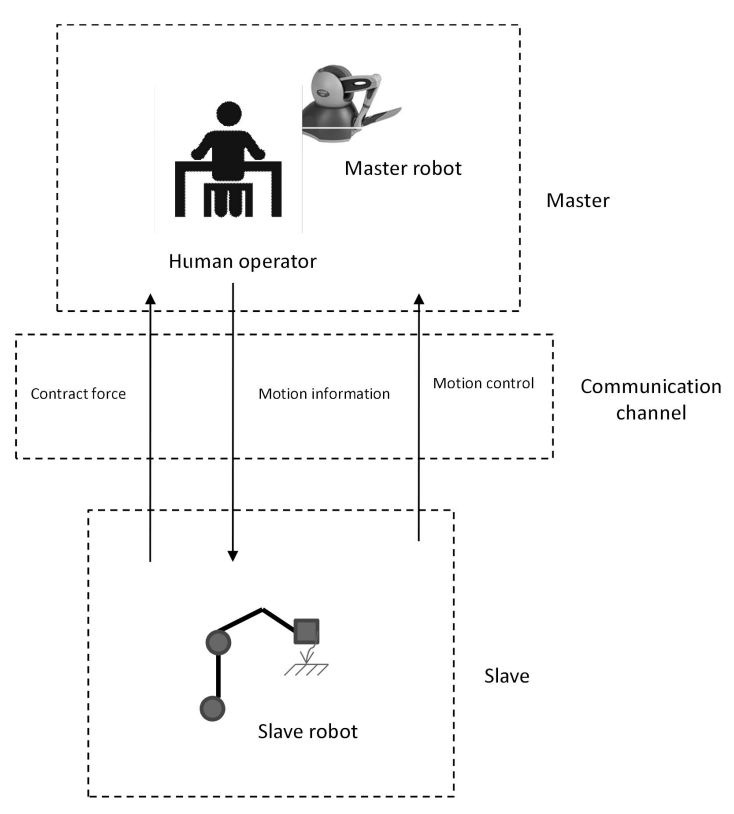

Fig. 2. Bilateral teleoperation with haptic feedback.

functional. Section 4 presents the transparency analysis in free motion and contact motion. In Section 5 simulations are reported to highlight the effectiveness of the novel bilateral teleoperation model and the control approach. Concluding remarks are given in Section 6 .

\section{Delayed bilateral teleoperation with haptic feedback}

The dynamic model of the teleoperation system in the task space 1 is

$$
\begin{aligned}
M_{m}\left(x_{m}\right) \ddot{x}_{m}+C_{m}\left(x_{m}, \dot{x}_{m}\right) \dot{x}_{m}+g_{m} & =f_{m}^{*}-f_{h}, \\
M_{s}\left(x_{s}\right) \ddot{x}_{s}+C_{s}\left(x_{s}, \dot{x}_{s}\right) \dot{x}_{s}+g_{s} & =f_{e}-f_{s}^{*} .
\end{aligned}
$$

According to the bilateral teleoperation, the model of the master robot should be replaced by the human operator model, see Fig. 2] Therefore, the bilateral teleoperation system based on admittance human operator model in the task space is

$$
\begin{aligned}
M_{a} \ddot{x}_{m}+B_{a} \dot{x}_{m}+K_{a} x_{m} & =f_{m}^{*}-f_{h}, \\
M_{s}\left(x_{s}\right) \ddot{x}_{s}+C_{s}\left(x_{s}, \dot{x}_{s}\right) \dot{x}_{s}+g_{s} & =f_{e}-f_{s}^{*},
\end{aligned}
$$

where $\ddot{x}_{i}, \dot{x}_{i}, x_{i}, i=m, s$, are the acceleration, the velocity and the end-effector position of the master and slave robots, $f_{h}, f_{e}$ are the operator and environment

\footnotetext{
${ }^{1}$ The task space (or Cartesian space) is defined by the position and orientation of the end effector of a robot.
}

forces, respectively. $f_{m}^{*}, f_{s}^{*}$ represent the control force inputs precompensated by the gravitational force and stiffness. $M_{a}, B_{a}$ and $K_{a}$ are the inertia, viscosity and stiffness constant positive matrices of human arm admittance, $M_{s}\left(x_{s}\right)$ is a symmetric and positive-definite inertia matrix, $C_{s}\left(x_{s}, \dot{x}_{s}\right)$ represent the Coriolis matrix of the slave system, and $g_{s}\left(x_{s}\right)$ is the vector of gravitational forces of the slave manipulator.

We use the following properties of the robotic model:

P1. The inertia matrix $M_{s}\left(x_{s}\right)$ is symmetric, positive definite and uniformly bounded by

$$
0<\lambda_{\min }\left\{M_{s}\right\} I \leq M_{s} \leq \lambda_{\max }\left\{M_{s}\right\} I<\infty
$$

with $\lambda_{\min }\left\{M_{s}\left(x_{s}\right)\right\}, \lambda_{\max }\left\{M_{s}\left(x_{s}\right)\right\} \in \mathbb{R}^{+}$, the minimum and maximum eigenvalues of $M_{s}\left(x_{s}\right)$, respectively.

$\mathrm{P} 2$. For the Coriolis matrix $C_{s}\left(x_{s}, \dot{x}_{s}\right)$, there exists a number $k_{c}>0$ such that

$$
\left\|C_{s}\left(x_{s}, \dot{x}_{s}\right)\right\| \leq k_{c}\left\|\dot{x}_{s}\right\|^{2}
$$

and $\dot{M}_{s}\left(x_{s}\right)-2 C_{s}\left(x_{s}, \dot{x}_{s}\right)$ is skew-symmetric, i.e.,

$$
\dot{x}_{s}^{T}\left[\dot{M}_{s}\left(x_{s}\right)-2 C_{s}\left(x_{s}, \dot{x}_{s}\right)\right] \dot{x}_{s}=0 .
$$

Teleoperation systems always have time delays in communication channels. We consider a variable time delay. Owing to the communication nature, it can not be negative and has a known upper bound $T_{i, \max }$, i.e.,

$$
0 \leq T_{i}(t) \leq T_{i, \max }<\infty, \quad i=m, s .
$$

For the task-space teleoperation, the system interacts with the human operator via the master robot, and with the environment via the end effector of the slave robot. We assume that the task-space interaction is passive. The assumption has been adopted by Lee and Spong (2006), Nuno et al. (2008) and Jafari et al. (2013). On the basis of the standard passivity notion (Lozano et al., 2007), there exist $\kappa_{i} \in \mathbb{R}^{+}$such that for all $t \geq 0$,

$$
\int_{0}^{t} \dot{x}_{m}^{T} f_{h} \mathrm{~d} \sigma \geq-\kappa_{m}, \quad-\int_{0}^{t} \dot{x}_{s}^{T} f_{e} \mathrm{~d} \sigma \geq-\kappa_{s}
$$

In order to simplify some calculations and focus on the main idea, we assume that the gravitational force and stiffness from the master and the slave models are precompensated by the controllers $f_{i}^{*}$, i.e., $f_{m}^{*}=f_{m}+g_{m}$ and $f_{s}^{*}=f_{s}+K_{a} x_{s}$, respectively. Hence, the dynamic model (1) changes to

$$
\begin{aligned}
M_{a} \ddot{x}_{m}+B_{a} \dot{x}_{m} & =f_{m}-f_{h}, \\
M_{s}\left(x_{s}\right) \ddot{x}_{s}+C_{s}\left(x_{s}, \dot{x}_{s}\right) \dot{x}_{s} & =f_{e}-f_{s} .
\end{aligned}
$$

Owing to lack of information about the variation in human and environment forces, we assume that the variation rate of these forces is bounded, i.e., $f_{h}, f_{e} \in \mathcal{L}_{\infty}$.

We expect to achieve the following control goals: 
1. The bilateral teleoperation system is stable if the human operator and the environment are passive and their forces are bounded.

2. The master-slave kinematics in the free-motion case satisfy

$$
\begin{aligned}
x_{m}-x_{s}\left(t-T_{s}(t)\right) & \rightarrow 0, \\
x_{s}-x_{m}\left(t-T_{m}(t)\right) & \rightarrow 0
\end{aligned}
$$

as $t \rightarrow \infty$.

3. The master-slave positions and forces in the contact motion case meet the requirement

$$
\begin{aligned}
x_{m}-x_{s}\left(t-T_{s}(t)\right) & \rightarrow 0, \\
x_{s}-x_{m}\left(t-T_{m}(t)\right) & \rightarrow 0, \\
f_{h}-f_{e}\left(t-T_{s}(t)\right) & \rightarrow 0 .
\end{aligned}
$$

This is transparency.

In order to achieve the control goals described above, we propose the following sliding mode PD control law for the delayed bilateral teleoperation system:

$$
\begin{aligned}
f_{m}= & -P\left(x_{m}-x_{s}\left(t-T_{s}(t)\right)\right)-B_{m} \dot{x}_{m} \\
& -\alpha \operatorname{sgn}\left(\dot{x}_{m}\right) f_{h e}^{T} f_{h e} \\
f_{s}= & P\left(x_{s}-x_{m}\left(t-T_{m}(t)\right)\right)+B_{s} \dot{x}_{s},
\end{aligned}
$$

where $\alpha>0$, the sliding mode term $\alpha \operatorname{sgn}\left(\dot{x}_{m}\right)$ is used to cancel the uncertainties in the teleoperation system, the PD control gains $P$ and $B_{i}(i=m, s)$ are positive definite matrices, $T_{m}(t)$ and $T_{s}(t)$ are the forward and backward communication time delays. Here

$$
f_{h e}=f_{h}-f_{e}\left[t-T_{s}(t)\right] .
$$

In addition, we assume that the upper bound of the round-trip delay $T_{r, \max }=T_{m, \max }+T_{s, \max }$ is known as a prior.

\section{Stability of nonlinear bilateral teleoperation with force feedback}

Stability is one of main bilateral teleoperation objectives; see the work of Lawrence (1993) for more detail. The original idea comes from the passivity of the teleoperation system (Hogan, 1985), where it is required that the human operator and the environment exhibit passive features.

According to the model dynamic of (7) as well as the control law (8), the stability of the closed-loop system is given in the following theorem.

Theorem 1. The nonlinear bilateral teleoperation system (7) is controlled by the controller (8). It is stable if $Q \geq 0$, where $Q$ is defined as

$$
\begin{aligned}
& Q=\left[\begin{array}{cc}
Q_{m} & 0 \\
0 & Q_{s}
\end{array}\right], \\
& Q_{m}=B_{m}+B_{a}-T_{r, \max } P, \\
& Q_{s}=B_{s}-T_{r, \max } P .
\end{aligned}
$$

Proof. Consider a nonnegative Lyapunov-Krasovskii functional,

$$
V(t)=\sum_{k=1}^{5} V_{k}(t)
$$

where

$$
\begin{aligned}
& V_{1}(t)=\frac{1}{2} \dot{x}_{m}^{T} M_{a} \dot{x}_{m}+\frac{1}{2} \dot{x}_{s}^{T} M_{s}\left(x_{s}\right) \dot{x}_{s}, \\
& V_{2}(t)=\frac{1}{2}\left(x_{m}-x_{s}\right)^{T} P\left(x_{m}-x_{s}\right), \\
& V_{3}(t)=\sum_{i=m, s}\left[\int_{-T_{i, \max }}^{0} \int_{t+\theta}^{t} \dot{x}_{i}^{T} P \dot{x}_{i} \mathrm{~d} \sigma \mathrm{d} \theta\right], \\
& V_{4}(t)=\int_{0}^{t}\left(\dot{x}_{m}^{T} f_{h}-\dot{x}_{s}^{T} f_{e}\right) \mathrm{d} \sigma+\kappa_{m}+\kappa_{s}, \\
& V_{5}(t)=\alpha \int_{0}^{t} \dot{x}_{m} \operatorname{sgn}\left(\dot{x}_{m}\right) f_{h e}^{T} f_{h e} \mathrm{~d} \theta .
\end{aligned}
$$

In the Lyapunov-Krasovskii functional, $V_{1}(t)$ represents the kinetic energy of the master and slave robots, $V_{2}(t)$ corresponds to the energy stored in the proportional control term, $V_{3}(t)$ is related to the network delay, $V_{4}(t)$ includes the energies input by the operator and environment and $V_{5}(t)$ corresponds to the force control term.

If we apply (4) in Property II to the teleoperation dynamic system (7), the time derivative of $V_{1}$ is simplified as

$$
\dot{V}_{1}(t)=\dot{x}_{m}^{T}\left(f_{m}-f_{h}-B_{a} \dot{x}_{m}\right)+\dot{x}_{s}^{T}\left(f_{e}-f_{s}\right) .
$$

Adding $\quad \dot{x}_{m} P\left(x_{s}\left(t-T_{s}(t)\right)-x_{s}\left(t-T_{s}(t)\right)\right) \quad$ and $\dot{x}_{s} P\left(x_{m}\left(t-T_{m}(t)\right)-x_{m}\left(t-T_{m}(t)\right)\right)$ to the time derivative of 111 results in

$$
\begin{aligned}
\dot{V}_{2}(t)= & -\dot{x}_{m} P \int_{t-T_{s}(t)}^{t} \dot{x}_{s} \mathrm{~d} \theta-\dot{x}_{s} P \int_{t-T_{m}(t)}^{t} \dot{x}_{m} \mathrm{~d} \theta \\
& +\dot{x}_{m} P\left(x_{m}-x_{s}\left(t-T_{s}(t)\right)\right) \\
& +\dot{x}_{s} P\left(x_{s}-x_{m}\left(t-T_{m}(t)\right)\right) .
\end{aligned}
$$

After some algebra the time derivative of (12) can be expressed as

$$
\dot{V}_{3}(t) \leq \sum_{i=m, s}\left[T_{i, \max } \dot{x}_{i}^{T} P \dot{x}_{i}-\int_{t-T_{i}(t)}^{t} \dot{x}_{i}^{T} P \dot{x}_{i} \mathrm{~d} \theta\right] .
$$

Applying the inequalities

$$
\begin{aligned}
&-\dot{x}_{m} P \int_{t-T_{s}(t)}^{t} \dot{x}_{s} \mathrm{~d} \theta-\int_{t-T_{s}(t)}^{t} \dot{x}_{s}^{T} P \dot{x}_{s} \mathrm{~d} \theta \\
& \leq T_{s, \max } \dot{x}_{m}^{T} P \dot{x}_{m}
\end{aligned}
$$


and

$$
\begin{aligned}
-\dot{x}_{s} P \int_{t-T_{m}(t)}^{t} \dot{x}_{m} \mathrm{~d} \theta-\int_{t-T_{m}(t)}^{t} & \dot{x}_{m}^{T} P \dot{x}_{m} \mathrm{~d} \theta \\
\leq & T_{m, \max } \dot{x}_{s}^{T} P \dot{x}_{s}
\end{aligned}
$$

the sum $\dot{V}_{2}(t)+\dot{V}_{3}(t)$ from Eqns. (16) and (17) is simplified as

$$
\begin{aligned}
\dot{V}_{23}(t) \leq & T_{r, \max }\left(\sum_{i=m, s} \dot{x}_{i}^{T} P \dot{x}_{i}\right) \\
& +\dot{x}_{m} P\left(x_{m}-x_{s}\left(t-T_{s}(t)\right)\right) \\
& +\dot{x}_{s} P\left(x_{s}-x_{m}\left(t-T_{m}(t)\right)\right) .
\end{aligned}
$$

Therefore, the time derivatives of (13) and (14) are

$$
\dot{V}_{4}(t)=\dot{x}_{m}^{T} f_{h}-\dot{x}_{s} f_{e}
$$

and

$$
\dot{V}_{5}(t)=\alpha \dot{x}_{m} \operatorname{sgn}\left(\dot{x}_{m}\right) f_{h e}^{T} f_{h e},
$$

respectively.

The sum $\dot{V}(t)=\sum_{k=1}^{5} \dot{V}_{k}(t)$, given by (15) and (18)-20) can be written as

$$
\begin{aligned}
\dot{V}(t) \leq & \dot{x}_{m}^{T}\left(f_{m}-f_{h}-B_{a} \dot{x}_{m}\right)+\dot{x}_{s}^{T}\left(f_{e}-f_{s}\right) \\
& +T_{r, \max }\left(\sum_{i=m, s} \dot{x}_{i}^{T} P \dot{x}_{i}\right)+\dot{x}_{m}^{T} f_{h}-\dot{x}_{s} f_{e} \\
& +\dot{x}_{m} P\left(x_{m}-x_{s}\left(t-T_{s}(t)\right)\right) \\
& +\dot{x}_{s} P\left(x_{s}-x_{m}\left(t-T_{m}(t)\right)\right) \\
& +\alpha \dot{x}_{m} \operatorname{sgn}\left(\dot{x}_{m}\right) f_{h e}^{T} f_{h e} .
\end{aligned}
$$

Using the control law $(8)$, we reduce the last equation to

$$
\dot{V}(t) \leq-\dot{x}^{T} Q \dot{x} \leq 0,
$$

where $Q=\operatorname{diag}\left\{Q_{m}, Q_{s}\right\} \geq 0$, such that $Q_{m}=B_{m}+$ $B_{a}-T_{r, \max } P$ and $Q_{s}=B_{s}-T_{r, \max } P$. If $Q \geq 0$ we can guarantee the boundedness of the Lyapunov-Krasovskii functional. Therefore, we can conclude that

$$
\dot{x}_{m} \in \mathcal{L}_{\infty}, \quad \dot{x}_{s} \in \mathcal{L}_{\infty}, \quad x_{m}-x_{s} \in \mathcal{L}_{\infty} .
$$

According to the above discussion, the control system is stable in the presence of time delay in communication channels.

\section{Transparency of nonlinear bilateral teleoperation}

The transparency is another major goal in teleoperation systems. When the teleoperation has haptic feedback, the transparency includes positions and forces between the master and slave robots (Yokokohji and Yoshikawa, 1994;
Salcudean et al., 2000), and the impedances perceived by the operator and the environment (Lawrence, 1993).

Both the human operator and the environment are in contact with the master and slave robots; see Fig. 2. The conditions for kinematic correspondence $x_{m} \equiv x_{s}$ as well as for force matching are

$$
\left.f_{\text {te }}\right|_{f_{h}^{*}=0}=f_{h},\left.\quad f_{t o}\right|_{f_{e}^{*}=0}=f_{e},
$$

where $f_{\text {te }}$ and $f_{\text {to }}$ are the forces transmitted by the human operator and the environment, and $f_{h}^{*}$ and $f_{e}^{*}$ are the operator and environment exogenous force inputs.

If the time delay in the communication channel is negligible, the performances of the position and force between the master and slave robots are prefect. In practice, the delays affect the velocities generated by the operator or caused by the environment. Therefore we need

$$
\begin{aligned}
x_{m}-x_{s}\left(t-T_{s}(t)\right) & \rightarrow 0, \\
f_{h}-f_{e}\left(t-T_{s}(t)\right) & \rightarrow 0,
\end{aligned}
$$

where $f_{h}^{*}=0$, and

$$
\begin{aligned}
x_{s}-x_{m}\left(t-T_{m}(t)\right) & \rightarrow 0, \\
f_{e}-f_{h}\left(t-T_{m}(t)\right) & \rightarrow 0
\end{aligned}
$$

for $f_{e}^{*}=0$.

For the four channels the bilateral teleoperation system (see Fig. 1), we use the following three-channel scheme, where there are no environment exogenous forces $\left(f_{e}^{*}=0\right)$. The transparency conditions are

$$
\begin{aligned}
x_{m}-x_{s}\left(t-T_{s}(t)\right) & \rightarrow 0, \\
f_{h}-f_{e}\left(t-T_{s}(t)\right) & \rightarrow 0
\end{aligned}
$$

for $f_{h}^{*}=0$, and

$$
x_{s}-x_{m}\left(t-T_{m}(t)\right) \rightarrow 0
$$

for $f_{e}^{*}=0$.

4.1. Free motion without force feedback. We assume the operator and environment forces to be equal zero, i.e., $f_{h}=f_{e}=0$.

Theorem 2. The position error in free motion converges to zero with respect to the control scheme (8), i.e.,

$$
\begin{aligned}
x_{m}-x_{s}\left(t-T_{s}(t)\right) & \rightarrow 0, \quad t \rightarrow \infty, \\
x_{s}-x_{m}\left(t-T_{m}(t)\right) & \rightarrow 0, \quad t \rightarrow \infty .
\end{aligned}
$$

Thus the transparency in kinematics is achieved.

Proof. In stability analysis, we conclude that $\dot{x}_{m}, \dot{x}_{s}$ and $x_{m}-x_{s}$ are bounded. Integrating both the sides of (22), we get the following expression:

$$
V(t)-V(0) \leq-\int_{0}^{t} \dot{x}^{T} Q \dot{x} \mathrm{~d} \theta .
$$


It can be rewritten as

$$
\int_{0}^{t} \dot{x}^{T} Q \dot{x} \mathrm{~d} \theta \leq V(0)-V(t) \leq V(0)<+\infty .
$$

This means

$$
\dot{x}_{i} \in \mathcal{L}_{2} .
$$

With the condition $Q \geq 0$, we can conclude that

$$
\dot{x}_{i} \in \mathcal{L}_{2} \cap \mathcal{L}_{\infty}
$$

Hence $\dot{x}_{i}$ is uniformly continuous and will converge to zero. From the equation

$$
\begin{aligned}
x_{m}-x_{s}\left(t-T_{s}(t)\right)= & x_{m}-x_{s}+x_{s} \\
& -x_{s}\left(t-T_{s}(t)\right)
\end{aligned}
$$

we get

$$
\begin{aligned}
x_{s}-x_{s}\left(t-T_{s}(t)\right) & =\int_{0}^{T_{s}(t)} \dot{x}_{s}(t+\theta) \mathrm{d} \theta \\
& \leq T_{s, \max }^{1 / 2}\left\|\dot{x}_{s}\right\|_{2}
\end{aligned}
$$

after applying the Schwartz inequality. Using (25) and (26), we deduce that

$$
x_{m}-x_{s}\left(t-T_{s}(t)\right) \in \mathcal{L}_{\infty} .
$$

With the same computations, we obtain

$$
x_{s}-x_{m}\left(t-T_{m}(t)\right) \in \mathcal{L}_{\infty} .
$$

The teleoperation dynamic equation (7) and the proposed control low (8) with $f_{h}=f_{e}=0$ can be written as

$$
\begin{aligned}
\ddot{x}_{m}= & -M_{a}^{-1}\left[P\left(x_{m}-x_{s}\left(t-T_{s}(t)\right)\right)\right. \\
& \left.+B_{m} \dot{x}_{m}+B_{a} \dot{x}_{m}\right], \\
\ddot{x}_{s}= & -M_{s}^{-1}\left(x_{s}\right)\left[P\left(x_{s}-x_{m}\left(t-T_{m}(t)\right)\right)\right. \\
& \left.+B_{s} \dot{x}_{s}+C_{s}\left(x_{s}, \dot{x}_{s}\right) \dot{x}_{s}\right] .
\end{aligned}
$$

From (27), Properties P1 and P2, we can deduce that

$$
\ddot{x}_{i} \in \mathcal{L}_{\infty} .
$$

The position synchronization arrives if $\ddot{x} \rightarrow 0$ is proven. The time derivative of (27) is

$$
\begin{aligned}
\frac{\mathrm{d}}{\mathrm{d} t} \ddot{x}_{m}= & -M_{a}^{-1} \frac{\mathrm{d}}{\mathrm{d} t}\left[P\left(x_{m}-x_{s}\left(t-T_{s}(t)\right)\right)\right. \\
& \left.+B_{m} \dot{x}_{m}+B_{a} \dot{x}_{m}\right]
\end{aligned}
$$

and

$$
\begin{aligned}
\frac{\mathrm{d}}{\mathrm{d} t} \ddot{x}_{s}= & -\frac{\mathrm{d}}{\mathrm{d} t} M_{s}^{-1}\left(x_{s}\right)\left[P\left(x_{s}-x_{m}\left(t-T_{m}(t)\right)\right)\right. \\
& \left.+B_{s} \dot{x}_{s}+C_{s}\left(x_{m}, \dot{x}_{m}\right) \dot{x}_{s}\right] \\
& -M_{s}^{-1}\left(x_{s}\right) \frac{\mathrm{d}}{\mathrm{d} t}\left[P\left(x_{s}-x_{m}\left(t-T_{m}(t)\right)\right)\right. \\
& \left.+B_{s} \dot{x}_{s}+C_{s}\left(x_{m}, \dot{x}_{m}\right) \dot{x}_{s}\right] .
\end{aligned}
$$

The first term from (29) is

$$
\begin{aligned}
\frac{\mathrm{d}}{\mathrm{d} t} M_{s}^{-1}\left(x_{s}\right)= & -M_{s}^{-1}\left(x_{s}\right) \dot{M}_{s}\left(x_{s}\right) M_{s}^{-1}\left(x_{s}\right) \\
= & -M_{s}^{-1}\left(x_{s}\right)\left[C_{s}\left(x_{s}, \dot{x}_{s}\right)\right. \\
& \left.+C_{s}^{T}\left(x_{s}, \dot{x}_{s}\right)\right] M_{s}^{-1}\left(x_{s}\right) .
\end{aligned}
$$

By using Properties $\mathrm{P} 1$ and $\mathrm{P} 2, \mathrm{~d} M_{s}^{-1} / \mathrm{d} t$ is bounded. Evidently (28) and 29) are bounded. In consequence,

$$
(\mathrm{d} / \mathrm{d} t) \ddot{x}_{i} \in \mathcal{L}_{\infty} .
$$

Therefore $\ddot{x}_{i}$ are uniformly continuous. Hence

$$
\int_{0}^{t} \ddot{x}_{i} \mathrm{~d} \theta=\dot{x}_{i}(t)-\dot{x}_{i}(0) .
$$

Since $\dot{x}_{i} \rightarrow 0, \int_{0}^{\infty} \ddot{x}_{i} \mathrm{~d} \theta$ is bounded. Using Barbalat' lemma, we conclude that

$$
\ddot{x}_{i} \rightarrow 0 \text {. }
$$

Owing to

$$
\dot{x}_{i} \rightarrow 0, \quad \ddot{x}_{i} \rightarrow 0
$$

the master and slave position synchronization is achieved

$$
\begin{aligned}
\lim _{t \rightarrow \infty} x_{m}-x_{s}\left(t-T_{s}(t)\right) & \rightarrow 0, \\
\lim _{t \rightarrow \infty} x_{s}-x_{m}\left(t-T_{m}(t)\right) & \rightarrow 0 .
\end{aligned}
$$

Accordingly, the position error converges to zero in free motion.

4.2. Motion with contract forces. In this case, the operator and environment forces are assumed to be bounded, i.e., $f_{h} \in \mathcal{L}_{\infty}$ and $f_{e} \in \mathcal{L}_{\infty}$.

Theorem 3. The position and force errors in the teleoperation system converge to zero with the control scheme (8) as $t \rightarrow \infty$, i.e.,

$$
\begin{aligned}
x_{m}-x_{s}\left(t-T_{s}(t)\right) & \rightarrow 0, \\
x_{s}-x_{m}\left(t-T_{m}(t)\right) & \rightarrow 0, \\
f_{h}-f_{e}\left(t-T_{s}(t)\right) & \rightarrow 0 .
\end{aligned}
$$

Proof. Since the gravity $g_{s}\left(x_{s}\right)$ and the stiffness $K_{a} x_{m}$ are bounded, and the forces are assumed to be bounded, it is possible to assume that $f_{m}$ and $f_{s}$ in (8) are also bounded. The teleoperation dynamic equation (7) and the proposed law control 8 can be rewritten as

$$
\begin{aligned}
\ddot{x}_{m}= & -M_{a}^{-1}\left[P\left(x_{m}-x_{s}\left(t-T_{s}(t)\right)\right)+B_{m} \dot{x}_{m}\right. \\
& \left.+B_{a} \dot{x}_{m}+f_{h}+\alpha \operatorname{sgn}\left(\dot{x}_{m}\right) f_{h e}^{T} f_{h e}\right], \\
\ddot{x}_{s}= & -M_{s}^{-1}\left(x_{s}\right)\left[P\left(x_{s}-x_{m}\left(t-T_{m}(t)\right)\right)\right. \\
& \left.+B_{s} \dot{x}_{s}-f_{e}+C_{s}\left(x_{s}, \dot{x}_{s}\right) \dot{x}_{s}\right] .
\end{aligned}
$$


Since $\dot{x}_{i} \in \mathcal{L}_{2} \cap \mathcal{L}_{\infty}, x_{m}-x_{s}\left(t-T_{s}(t)\right), x_{s}-$ $x_{m}\left(t-T_{m}(t)\right) \in \mathcal{L}_{\infty}, f_{h}, f_{e} \in \mathcal{L}_{\infty}$, using Properties $\mathrm{P} 1$ and $\mathrm{P} 2$, we can conclude that

$$
\ddot{x}_{m} \in \mathcal{L}_{\infty}, \quad \ddot{x}_{s} \in \mathcal{L}_{\infty} .
$$

Therefore, due to Barbalat's lemma,

$$
\dot{x}_{i} \rightarrow 0 \text {. }
$$

The time derivative of $(30)$ is

$$
\begin{aligned}
\frac{\mathrm{d}}{\mathrm{d} t} \ddot{x}_{m}= & -M_{a}^{-1} \frac{\mathrm{d}}{\mathrm{d} t}\left[P\left(x_{m}-x_{s}\left(t-T_{s}(t)\right)\right)\right. \\
& +B_{m} \dot{x}_{m}+B_{a} \dot{x}_{m}+f_{h} \\
& \left.+\alpha \operatorname{sgn}\left(\dot{x}_{m}\right) f_{h e}^{T} f_{h e}\right],
\end{aligned}
$$

and

$$
\begin{aligned}
\frac{\mathrm{d}}{\mathrm{d} t} \ddot{x}_{s}= & -\frac{\mathrm{d}}{\mathrm{d} t} M_{s}^{-1}\left(x_{s}\right)\left[P\left(x_{s}-x_{m}\left(t-T_{m}(t)\right)\right)\right. \\
& \left.+B_{s} \dot{x}_{s}-f_{e}+C_{s}\left(x_{s}, \dot{x}_{s}\right) \dot{x}_{s}\right] \\
& -M_{s}^{-1}\left(x_{s}\right) \frac{\mathrm{d}}{\mathrm{d} t}\left[P\left(x_{s}-x_{m}\left(t-T_{m}(t)\right)\right)\right. \\
& \left.+B_{s} \dot{x}_{s}-f_{e}+C_{s}\left(x_{s}, \dot{x}_{s}\right) \dot{x}_{s}\right] .
\end{aligned}
$$

The first term of (32) is

$$
\begin{aligned}
\frac{\mathrm{d}}{\mathrm{d} t} M_{s}^{-1}\left(x_{s}\right)= & -M_{s}^{-1}\left(x_{s}\right) \dot{M}_{s}\left(x_{s}\right) M_{s}^{-1}\left(x_{s}\right) \\
= & -M_{s}^{-1}\left(x_{s}\right)\left[C_{s}\left(x_{s}, \dot{x}_{s}\right)\right. \\
& \left.+C_{s}^{T}\left(x_{s}, \dot{x}_{s}\right)\right] M_{s}^{-1}\left(x_{s}\right) .
\end{aligned}
$$

By using Properties $\mathrm{P} 1$ and $\mathrm{P} 2, \mathrm{~d} M_{s}^{-1} / \mathrm{d} t$ are bounded. Evidently, (31) and (32) are bounded. In consequence, $(\mathrm{d} / \mathrm{d} t) \ddot{x}_{i} \in \mathcal{L}_{\infty}$. Therefore $\ddot{x}_{i}$ are uniformly continuous. Using Barbalat's lemma, we can conclude that

$$
\ddot{x}_{i} \rightarrow 0 \text {. }
$$

As $\dot{x}_{i} \rightarrow 0$ and $\ddot{x}_{i} \rightarrow 0$ in the dynamic slave teleoperation system, we get

$$
\begin{aligned}
& P\left(x_{s}-x_{m}\left(t-T_{m}(t)\right)\right)-f_{e} \rightarrow 0, \\
& P\left(x_{m}-x_{s}\left(t-T_{s}(t)\right)\right)+f_{h} \rightarrow 0 .
\end{aligned}
$$

Consequently,

$$
\begin{aligned}
x_{s}-x_{m}\left(t-T_{m}(t)\right) & \rightarrow 0, \\
x_{m}-x_{s}\left(t-T_{s}(t)\right) & \rightarrow 0 .
\end{aligned}
$$

Now we apply the last results, i.e.,

$$
\begin{aligned}
x_{i} & \rightarrow 0 \\
x_{m}-x_{s}\left(t-T_{s}(t)\right) & \rightarrow 0, \\
x_{m}-x_{s}\left(t-T_{s}(t)\right) & \rightarrow 0 .
\end{aligned}
$$

The teleoperation system (7) with the control law (8) are simplified

$M_{a} \ddot{x}_{m}=\varepsilon\left(f_{h}-f_{e}\left(t-T_{s}(t)\right)\right)^{T}\left(f_{h}-f_{e}\left(t-T_{s}(t)\right)\right)$

Premultiplying both the sides by $\varepsilon^{T} M_{a}^{-1}$, we get

$$
\begin{aligned}
\varepsilon^{T} \ddot{x}_{m}= & \varepsilon^{T} M_{a}^{-1} \varepsilon\left(f_{h}-f_{e}\left(t-T_{s}(t)\right)\right)^{T} \\
& \cdot\left(f_{h}-f_{e}\left(t-T_{s}(t)\right)\right) .
\end{aligned}
$$

Using Property $\mathrm{P} 1$ and $1 /\left(\lambda_{\max }\left\{M_{a}\right\}\right) I \leq M_{a}^{-1}$, we have

$$
\begin{aligned}
\varepsilon^{T} \ddot{x}_{m} \geq & \frac{1}{\lambda_{\max }\left\{M_{a}\right\}}\|\varepsilon\|_{2}^{2}\left(f_{h}-f_{e}\left(t-T_{s}(t)\right)\right)^{T} \\
& \cdot\left(f_{h}-f_{e}\left(t-T_{s}(t)\right)\right) .
\end{aligned}
$$

Note that $\left(f_{h}-f_{e}\left(t-T_{s}(t)\right)\right)^{T}\left(f_{h}-f_{e}\left(t-T_{s}(t)\right)\right)$ and $\|\varepsilon\|_{2}^{2}$ are nonnegative, and $\lambda_{\max }\left\{M_{a}\right\}$ is positive.

If $\varepsilon^{T} \ddot{x}_{m}=0$, we have

$$
f_{h}-f_{e}\left(t-T_{s}(t)\right)=0
$$

and the proof is completed.

If $\varepsilon^{T} \ddot{x}_{m}>0$, in view of the fact that all the elements of $\varepsilon^{T}$ are positive, there exist positive $\ddot{x}_{m_{i}}$ as $t \rightarrow \infty$,

$$
\sum_{i=1}^{n} \ddot{x}_{m_{i}}>0
$$

But this is a contradiction with $\dot{x}_{m} \rightarrow 0$. Therefore $\varepsilon^{T} \ddot{x}_{m}$ tends to zero and $f_{h}-f_{e}\left(t-T_{s}(t)\right) \rightarrow 0$. The tracking error of the force converges to zero.

\section{Simulations}

In order to verify the proposed theoretical results, the slave manipulator is considered to be a 2-DOF planar robot with revolute joints. The slave manipulator dynamics have the following inertia matrix (Lee and $\mathrm{Li}, 2005$ )

$$
M_{s}\left(q_{s}\right)=\left[\begin{array}{cc}
\alpha+2 \beta \cos \left(q_{2}\right) & \delta+\beta \cos \left(q_{2}\right) \\
\delta+\beta \cos \left(q_{2}\right) & \delta
\end{array}\right]
$$

where $q_{k}$ is the articular position of each link with $k \in$ $1,2, \alpha=l_{2}^{2} m_{2}+l_{1}^{2}\left(m_{1}+m_{2}\right), \beta=l_{1} l_{2} m_{2}$, and $\delta=l_{2}^{2} m_{2}$. The lengths for both links $l_{1}$ and $l_{2}$ in the manipulator are $0.5 \mathrm{~m}$, for simplicity. The masses for each link correspond to $m_{1}=3.24 \mathrm{~kg}$, and $m_{2}=0.31 \mathrm{~kg}$, respectively. Coriolis and centrifugal forces are modeled as the vector

$$
C_{s}\left(q_{s}, \dot{q}_{s}\right)=\left[\begin{array}{c}
-\beta \sin \left(q_{2}\right) \dot{q}_{2}^{2}-2 \beta \sin \left(q_{2}\right) \dot{q}_{1} \dot{q}_{2} \\
\beta \sin \left(q_{2}\right) \dot{q}_{1}^{2}
\end{array}\right],
$$

where $\dot{q}_{k}$ are the respective revolute velocities of the two links. The gravity effects are represented by

$$
g_{s}\left(q_{s}\right)=g\left[\begin{array}{c}
\frac{1}{l_{2}} \delta \cos \left(q_{1}+q_{2}\right)+\frac{1}{l_{1}}(\alpha-\delta) \cos \left(q_{1}\right) \\
\frac{1}{l_{2}} \delta \cos \left(q_{1}+q_{2}\right)
\end{array}\right] .
$$



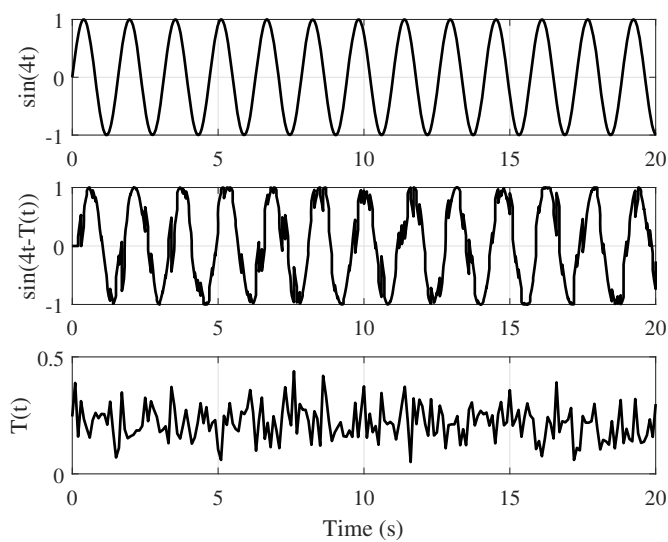

Fig. 3. Delayed signal with a variable time delay.

It should be clarified that the human exerts a force on the local manipulator's tip, and the remote manipulator interaction with the environment is also measured at its tip. Hence, for the simulations, we use the expression

$$
\tau_{e}=J_{s}^{T}\left(q_{s}\right) f_{e}
$$

where $J_{s}\left(q_{s}\right)$ is the Jacobian of the slave robot manipulator.

The admittance human operator model has the following inertia, stiffness and damping matrix gains (Lawrence, 1993)

$$
\begin{aligned}
M_{a} & =\operatorname{diag}\{17.5,17.5\} \mathrm{N}, \\
B_{a} & =\operatorname{diag}\{175,175\} \mathrm{N} / \mathrm{m}, \\
K_{a} & =\operatorname{diag}\{175,175\} \mathrm{Ns} / \mathrm{m} .
\end{aligned}
$$

The gains of the controller are $P=50 I, B_{i}=10 I$, where $I$ is the identity matrix, and $T_{i, \max }=0.45 \mathrm{~s}$, which clearly fulfills the stability condition. The initial positions for the local and remote manipulators are $x_{m}(0)=$ $[0,0]^{T}$ and $x_{s}(0)=[0,0]^{T}$, whereas the initial velocities are zero.

For simplicity, the variable time delays are the same for both forward and backward paths. Figure 3 shows how a sinusoidal signal is sent through variable time delay from the local robot to the remote robot.

5.1. Simulation for free motion. We first show that the position tracking errors (kinematic in free motion) converge, i.e.,

$$
\begin{aligned}
x_{m}-x_{s}\left(t-T_{s}(t)\right) & \rightarrow 0, \\
x_{s}-x_{m}\left(t-T_{m}(t)\right) & \rightarrow 0 .
\end{aligned}
$$

It is a free motion. The slave robot does not contact with the environment, so that $f_{e}=0$. The human's force $f_{h}$ applied to the master robot along the $x$ direction is shown

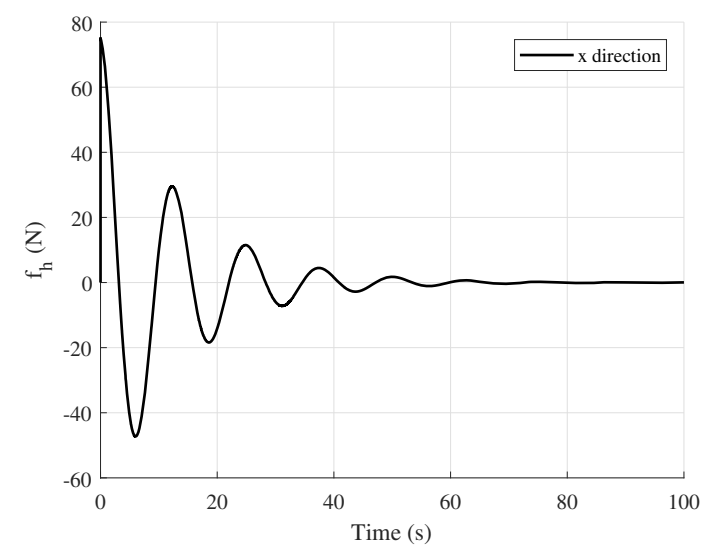

Fig. 4. Human force $f_{h}$ is applied to the master robot in the $x$ direction.

in Fig. 4. The force applied along the $y$ direction is null. After a while, $f_{h}$ decreases to zero. The position tracking of the master and the slave sites are shown in Fig. 5. We can see that the tracking errors converge to zero.

From the simulation, we can conclude that

- The bilateral teleoperation system is stable in free motion.

- Transparency (kinematic correspondence) is guaranteed.

- There is a good performance in free motion.

The following simulations will show that the bilateral teleoperation system with the control law can guarantee stability and transparency with contact forces.

5.2. Simulation for contact force. The simulations show that we can guarantee a stable bilateral teleoperation
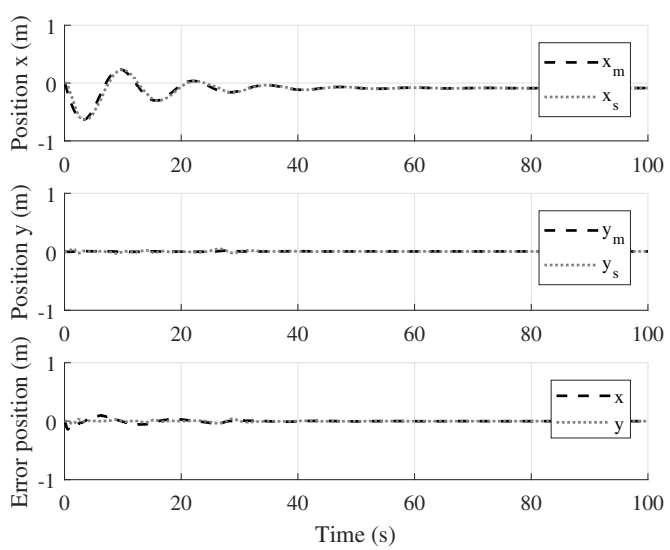

Fig. 5. Position tracking between the local and remote sites. 


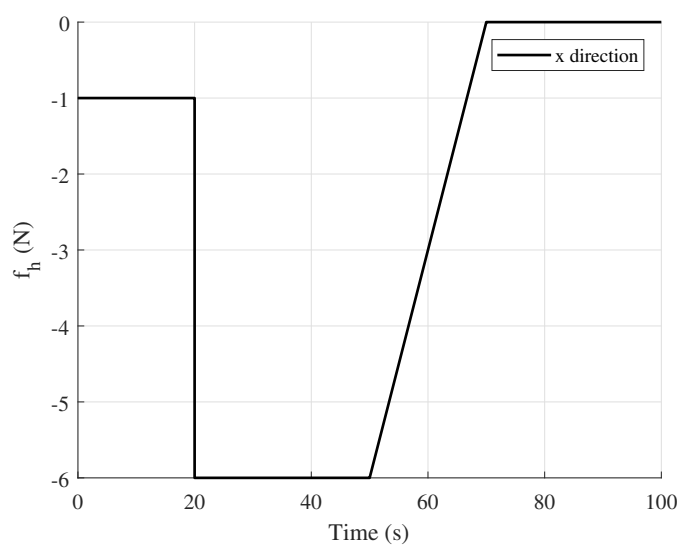

Fig. 6. Human's force $f_{h}$ is applied to the master robot in the $x$ direction.

in transition motion. Also, we improve the transparency in the three-channel architecture, i.e.,

$$
\begin{aligned}
x_{m}-x_{s}\left(t-T_{s}(t)\right) & \rightarrow 0, \\
x_{s}-x_{m}\left(t-T_{m}(t)\right) & \rightarrow 0, \\
f_{h}-f_{e}\left(t-T_{s}(t)\right) & \rightarrow 0
\end{aligned}
$$

In order to evaluate the contact force, we implement a wall in the slave environment at $0.5 \mathrm{~m}$. It is modeled as a spring-damper system along the $x$ direction. The spring and damping gains are $200 \mathrm{~N} / \mathrm{m}$ and $0.1 \mathrm{Ns} / \mathrm{m}$. There is no exogenous force in environment, that is, the wall is static. The force is applied by a human, see Fig. 6. For a period of $20 \mathrm{~s}$, the human operator stabilizes the master robot with $0.5 \mathrm{~N}$. In the time interval from 20 to $50 \mathrm{~s}$, the human pushes the master robot with the force $3 \mathrm{~N}$ to make a hard contact. Finally, in the time interval from 50 to $90 \mathrm{~s}$, the human retracts slowly until the zero force. The scenario is presented in Fig. 6 As in free motion simulation, we just apply force in the $x$ direction. In Fig. 7 we can see the position errors of the master and the slave sites.

Figure 8 shows the force correspondence. We can see that when the robot touches the wall, and transmits back the force to the human operator, our controller improves the control performances, and

$$
f_{h}-f_{e}\left(t-T_{s}(t)\right) \rightarrow 0 \text {. }
$$

This yields transparency.

The main problems in bilateral teleoperation systems with force feedback occur in contact motion and transition motion. We have the following remarks:

- Bilateral teleoperation system is stable in contact motion.

- Transparency (kinematic and force correspondences)
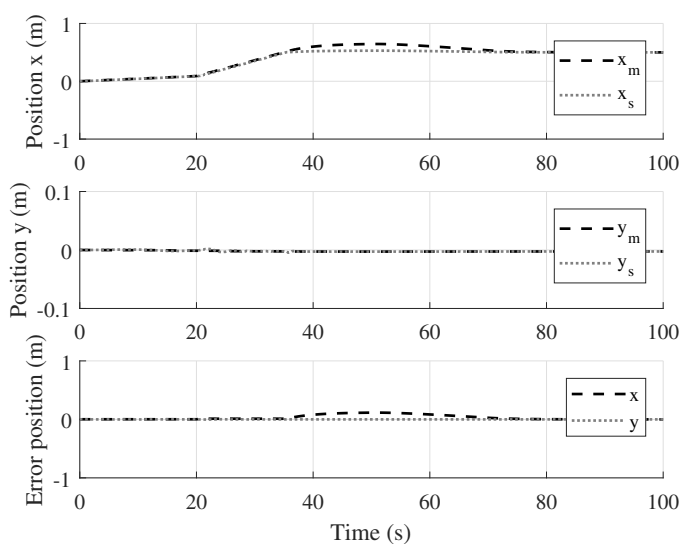

Fig. 7. Position tracking between the local and remote sites.

is guaranteed,

$$
\begin{aligned}
x_{m}-x_{s}\left(t-T_{s}(t)\right) & \rightarrow 0, \\
x_{s}-x_{m}\left(t-T_{m}(t)\right) & \rightarrow 0 .
\end{aligned}
$$

5.3. Discussion. The novel scheme for the bilateral teleoperation system based on a human model can improve transparency in free and contact motions. For delayed bilateral teleoperation with haptic feedback, we have the following remarks:

- Perfect transparency does not exist in bilateral teleoperation because of a time delay in the communication channel. But our control law in task space can improve it.

- The control law has a small gain. This gain allows us to reduce the force error. The quadratic error of the force depends on human and environment forces. When it is bigger, the quadratic force increases, and
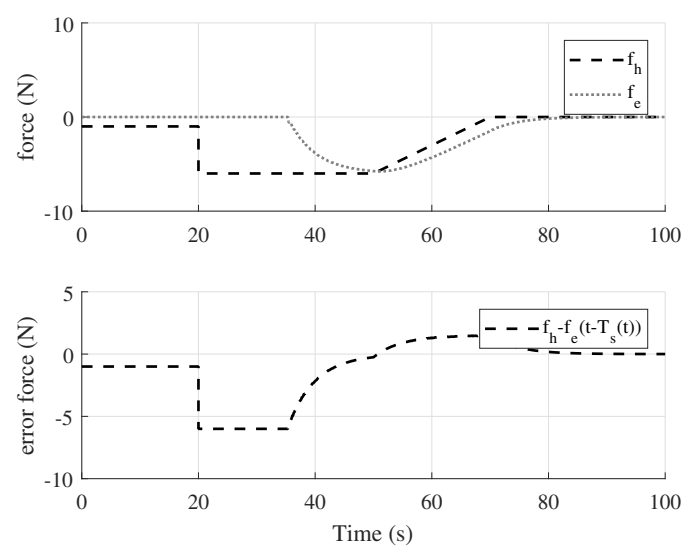

Fig. 8. Force tracking between the local and remote sites. 
a motion transition may happen. We consider a small fixed gain to avoid a large contact force, because we do not know the contact force. A variable gain would help us to improve the motion transition, such that it is increased in a smooth contact.

- The parameters of the human model are constant. But these parameters depend on the motion and the operators. Therefore, it is necessary to develop an adaptive algorithm.

- In most scenarios the environment is unknown. A force estimator on the slave side would help to improve the performance.

Stability analysis based on Lyapunov's method is less conservative than passivity-based approaches, although it still involves great challenges to be solved. In the control of nonlinear robots in teleoperation there is no mathematical proof for a PID control based on the Lyapunov method. In the position/force control it has not been shown yet that exponential stability can be achieved.

In this work, no specific kind of disturbances has been considered. Is well known that robotic systems are subject to different types of disturbances, such as inertial parameter variations, friction, dynamic uncertainties and noise that affect stability and transparency directly. In practical robotic applications, ideal dynamic models are impossible to derive, while sensors may introduce large noise, especially force sensors that amplify noise, which results in a noisy force signal transmitted via time-delay communication for control. In consequence, mounting force sensors on a robot yields some limitations. In order to overcome this problem, force observers have been gradually deployed in teleoperation systems. This topic will be considered in our scheme as future work.

\section{Conclusion}

This paper studies the stability and transparency of bilateral teleoperation. Teleoperation systems have nonlinear bilateral, time-varying time delays and force feedback. By using the Lyapunov-Krasovskii method and task space admittance control, the stability and transparency of a closed-loop system are guaranteed. The performance of the proposed control method is evidenced by simulations. It has been shown that the controller presented in this paper improves the performance of the bilateral teleoperation.

\section{References}

Anderson, R.J. and Spong, M.W. (1989). Bilateral control of teleoperators with time delay, IEEE Transactions on Automatic Control 34(5): 494-501.

Balachandran, R., Artigas, J., Mehmood, U. and Ryu, J. (2016). Performance comparison of wave variable transformation and time domain passivity approaches for time-delayed teleoperation: Preliminary results, 2016 IEEE/RSJ International Conference on Intelligent Robots and Systems (IROS), Daejeon, Korea, pp. 410-417.

Chen, Z., Huang, F., Song, W. and Zhu, S. (2018). A novel wave-variable based time-delay compensated four-channel control design for multilateral teleoperation system, IEEE Access 6(5): 25506-25516.

Chiaverini, S., Siciliano, B. and Villani, L. (1994). Force/position regulation of compliant robot manipulators, IEEE Transactions on Automatic Control 39(3): 647-652.

Cho, H.C. and Park, J.H. (2005). Stable bilateral teleoperation under a time delay using a robust impedance control, Mechatronics 15(5): 611 - 625 .

Chopra, N., Spong, M.W., Hirche, S. and Buss, M. (2003). Bilateral teleoperation over the internet: The time varying delay problem, American Control Conference, Denver, CO, USA, pp. 155-160.

Chopra, N., Spong, M. W. and Lozano, R. (2008). Synchronization of bilateral teleoperators with time delay, Automatica 44(8): 2142-2148.

Chopra, N., Spong, M.W., Ortega, R. and Barabanov, N.E. (2006). On tracking performance in bilateral teleoperation, IEEE Transactions on Robotics 22(4): 861-866.

Ehrampoosh, S., Dave, M., Kia, M.A., Rablau, C. and Zadeh, M.H. (2013). Providing haptic feedback in robot-assisted minimally invasive surgery: A direct optical force-sensing solution for haptic rendering of deformable bodies, Computer Aided Surgery 18(5-6): 129-141, DOI: 10.3109/10929088.2013.839744.

Erden, M.S. and Marić, B. (2011). Assisting manual welding with robot, Robotics and Computer-Integrated Manufacturing 27(4): 818-828.

Ganjefar, S., Rezaei, S. and Hashemzadeh, F. (2017). Position and force tracking in nonlinear teleoperation systems with sandwich linearity in actuators and time-varying delay, $\mathrm{Me}$ chanical Systems and Signal Processing 86(1): 308-324.

Hogan, N. (1985). Impedance control: An approach to manipulation. Part II: Implementation, Journal of Dynamic Systems, Measurement, and Control 107(1): 8-16, DOI: 10.1115/1.3140713.

Hokayem, P.F. and Spong, M.W. (2006). Bilateral teleoperation: An historical survey, Automatica 42(12): 2035-2057.

Imaida, T., Yokokohji, Y., Doi, T., Oda, M. and Yoshikawa, T. (2004). Ground-space bilateral teleoperation of ETS-VII robot arm by direct bilateral coupling under 7-s time delay condition, IEEE Transactions on Robotics and Automation 20(3): 499-511.

Ishii, T. and Katsura, S. (2012). Bilateral control with local force feedback for delay-free teleoperation, 12th IEEE International Workshop on Advanced Motion Control (AMC), Sarajevo, Bosnia and Herzegovina, pp. 1-6.

Jafari, A., Rezaei, S., Ghidary, S.S., Zareinejad, M., Baghestan, K. and Dehghan, M. (2013). A stable perturbation estimator in force-reflecting passivity-based teleoperation, Transactions of the Institute of Measurement and Control 35(2): 147-156, DOI: 10.1177/0142331211435849. 
Jordan, M.A. and Bustamante, J.L. (2007). On the presence of nonlinear oscillations in the teleoperation of underwater vehicles under the influence of sea wave and current, American Control Conference, New York City, NY, USA, pp. 894-899.

Kawashima, K., Tadano, K., Sankaranarayanan, G. and Hannaford, B. (2008). Model-based passivity control for bilateral teleoperation of a surgical robot with time delay, IEEE/RSJ International Conference on Intelligent Robots and Systems, Nice, France, pp. 1427-1432.

Lawrence, D.A. (1993). Stability and transparency in bilateral teleoperation, IEEE Transactions on Robotics and Automation 9(5): 624-637.

Lee, D. and Li, P.Y. (2005). Passive bilateral control and tool dynamics rendering for nonlinear mechanical teleoperators, IEEE Transactions on Robotics 21(5): 936-951.

Lee, D. and Spong, M.W. (2006). Passive bilateral teleoperation with constant time delay, IEEE Transactions on Robotics 22(2): 269-281.

Liu, S., Wei, X., Zheng, W. and Yang, B. (2018). A four-channel time domain passivity approach for bilateral teleoperator, IEEE International Conference on Mechatronics and Automation (ICMA), Changchun, China, pp. 318-322.

Lozano, R., Brogliato, B., Egeland, O. and Maschke, B. (2007). Dissipative Systems Analysis and Control, Springer, London.

Mobasser, F. and Hashtrudi-Zaad, K. (2008). Transparent rate mode bilateral teleoperation control, International Journal of Robotics Research 27(1): 57-72, DOI: 10.1177/0278364907083397.

Munir, S. and Book, W.J. (2002). Internet-based teleoperation using wave variables with prediction, IEEE/ASME Transactions on Mechatronics 7(2): 124-133.

Munir, S. and Book, W.J. (2003). Control techniques and programming issues for time delayed internet based teleoperation, Journal of Dynamic Systems, Measurement, and Control 125(2): 205-214, DOI: 10.1115/1.1568120.

Niemeyer, G. and Slotine, J.E. (1998). Towards force-reflecting teleoperation over the internet, IEEE International Conference on Robotics and Automation, Leuven, Belgium, Vol. 3 , pp. 1909-1915 vol.3.

Niemeyer, G. and Slotine, J.J.E. (1991). Stable adaptive teleoperation, IEEE Journal of Oceanic Engineering 16(1): 152-162.

Niemeyer, G. and Slotine, J.-J.E. (2004). Telemanipulation with time delays, International Journal of Robotics Research 23(9): 873-890, DOI: 10.1177/0278364904045563.

Nohmi, M. (2003). Space teleoperation using force reflection of communication time delay, 2003 IEEE/RSJ International Conference on Intelligent Robots and Systems (IROS 2003), Las Vegas, NV, USA, Vol. 3, pp. 2809-2814.

Nuno, E. and Basanez, L. (2009). Nonlinear bilateral teleoperation: Stability analysis, IEEE International Conference on Robotics and Automation, Kobe, Japan, pp. 3718-3723.
Nuno, E., Ortega, R., Barabanov, N. and Basaez, L. (2008). A globally stable pd controller for bilateral teleoperators, IEEE Transactions on Robotics 24(3): 753-758.

Nuno, E., Ortega, R. and Basanez, L. (2010). An adaptive controller for nonlinear teleoperators, Automatica 46(1): 155-159.

Ousaid, A.M., Haliyo, D.S., Rgnier, S. and Hayward, V. (2015). A stable and transparent microscale force feedback teleoperation system, IEEE/ASME Transactions on Mechatronics 20(5): 2593-2603.

Rebelo, J. and Schiele, A. (2015). Time domain passivity controller for 4-channel time-delay bilateral teleoperation, IEEE Transactions on Haptics 8(1): 79-89.

Salcudean, S.E., Zhu, M., Zhu, W.-H. and Hashtrudi-Zaad, K. (2000). Transparent bilateral teleoperation under position and rate control, International Journal of Robotics Research 19(12): 1185-1202, DOI: $10.1177 / 02783640022068020$.

Tanner, N.A. and Niemeyer, G. (2005). Improving perception in time delayed teleoperation, Proceedings of the 2005 IEEE International Conference on Robotics and Automation, Barcelona, Spain, pp. 354-359.

Wen, J.T. and Murphy, S. (1991). Stability analysis of position and force control for robot arms, IEEE Transactions on Automatic Control 36(3): 365-371.

Xu, X., Cizmeci, B., Schuwerk, C. and Steinbach, E. (2016). Model-mediated teleoperation: Toward stable and transparent teleoperation systems, IEEE Access 4(1): 425-449.

Yokokohji, Y., Imaida, T. and Yoshikawa, T. (1999). Bilateral teleoperation under time-varying communication delay, IEEE/RSJ International Conference on Intelligent Robots and Systems: Human and Environment Friendly Robots with High Intelligence and Emotional Quotients, Kyongju, South Korea, Vol. 3, pp. 1854-1859.

Yokokohji, Y. and Yoshikawa, T. (1994). Bilateral control of master-slave manipulators for ideal kinesthetic coupling-formulation and experiment, IEEE Transactions on Robotics and Automation 10(5): 605-620.

Zhu, M. and Salcudean, S.E. (1995). Achieving transparency for teleoperator systems under position and rate control, IEEE/RSJ International Conference on Intelligent Robots and Systems: Human Robot Interaction and Cooperative Robots, Pittsburgh, PA, USA, Vol. 2, pp. 7-12.

Edgar Estrada is a $\mathrm{PhD}$ student in the Automatic Control Department at Cinvestav-IPN. He received a degree in electronic engineering in 2014 and an MS degree in automatic control in 2015 from IPN. His research interests include time delay systems and teleoperation.

Wen Yu received his BS degree from Tsinghua University, Beijing, China, in 1990 and the MS and PhD degrees, both in electrical engineering, from Northeastern University, Shenyang, China, in 1992 and 1995 , respectively. Since 1996, he has been with National Polytechnic Institute (CINVESTAV-IPN), Mexico City, Mexico, where he is currently a professor and the chair of the Automatic Control Department. From 
2002 to 2003, he held research positions with the Mexican Institute of Petroleum. He was a senior visiting research fellow with Queen's University Belfast, UK, from 2006 to 2007, and a visiting associate professor with the University of California, Santa Cruz, USA, from 2009 to 2010. He has published more than 100 research papers. He is a member of the Mexican Academy of Sciences.

Xiaoou Li received the $\mathrm{BS}$ and the $\mathrm{PhD}$ degrees in applied mathematics and electrical engineering from Northeastern University, China, in 1991 and 1995, respectively. From 1995 to 1997, she was a lecturer of electrical engineering at the Department of Automatic Control of Northeastern University, China. From 1998 to 1999, she was an associate professor of computer science at Centro de Instrumentos-UNAM. Since 2000, she has been a professor of computer science at the Department of Electrical Engineering, CINVESTAV-IPN, México. Her research interests include Petri nets neural networks, advanced database systems, computer integrated manufacturing, and discrete event systems.

Received: 30 November 2018

Revised: 10 April 2019

Re-revised: 27 May 2019

Accepted: 28 July 2019 